\section{Epidemiologia e Saúde Coletiva no Brasil: desafios para a formação em pesquisa *}

\section{Epidemiology and Public Health in Brazil: challenges for research training}

\section{Estela Maria Aquino}

Programa de Pós-Graduação em Saúde Coletiva (PPGSC), Instituto de Saúde Coletiva, Universidade Federal da Bahia

* Apresentado no Seminário "Inquéritos populacionais: aspectos metodológicos, operacionais e éticos", promovido pela Comissão de Epidemiologia da ABRASCO, pela Faculdade de Saúde Pública (USP) e pelo Departamento de Medicina Social da Santa Casa de São Paulo, em 17 a 19 de abril de 2007. Correspondência: Estela Maria Aquino, Instituto de Saúde Coletiva, Rua Basílio da Gama, s/ no. - 2 andar, Campus Universitário do Canela, Salvador,BA CEP 40110-040, e-mail: estela@ufba.br

\section{Resumo}

A pesquisa epidemiológica tem crescido muito no Brasil, o que pode ser atribuído em parte à expansão da formação pós-graduada. Mudanças na avaliação dos programas, conjugando qualidade e eficiência, têm colocado desafios para a formação em pesquisa impulsionando o desenvolvimento de estratégias pedagógicas variadas. Neste artigo, pretendo debater a proposta de "consórcio de pesquisas" do Programa de Pós-Graduação em Epidemiologia da Universidade Federal de Pelotas, destacando algumas implicações pedagógicas deste modelo e apresentando em contraste a estratégia adotada pelo Programa de Pós-Graduação em Saúde Coletiva da Universidade Federal da Bahia para formação em pesquisa epidemiológica. Procuro contextualizar o debate situando-o brevemente no quadro da epidemiologia brasileira, suas principais características de desenvolvimento e pontuando o potencial dos inquéritos domiciliares como ferramenta de diagnóstico de saúde para subsidiar políticas públicas. Após discutir as principais vantagens e limites dos modelos adotados em dois importantes pólos de ensino e pesquisa epidemiológica no país, concluo levantando algumas questões que se colocam como desafios para formação pós-graduada de pesquisadores na atualidade.

Palavras-chave: Educação de pós-graduação. Programas de pós-graduação. Formação em pesquisa. Saúde coletiva. Métodos epidemiológicos. Estudos transversais. 
Abstract

Epidemiological research has been growing markedly in Brazil, which can be partly attributed to the expansion of graduate training. Changes in the evaluation of programs, combining quality and efficiency, have posed challenges for research training, driving the development of many teaching strategies. This paper intends to debate the proposal of the "research consortium" of the Graduate Epidemiology Program of Pelotas Federal University, highlighting some educational implications of this model and presenting, in contrast, the strategy adopted by the Graduate Collective Health Program of Bahia Federal University for training on epidemiological research. I have tried to set the context for the debate by outlining a brief background of Brazilian epidemiology, describing its main features in terms of development, and identifying the potential of home surveys as a health diagnosis tool to support public policies. After addressing the main advantages and limits of the models adopted in two important centers of epidemiological education and research in Brazil, I conclude by raising some issues that are challenges for graduate research education today.

Keywords: Education. Graduate. Graduate programs. Research training. Public health. Epidemiologic methods. Cross-sectional studies.

\section{Introdução}

A pesquisa em saúde no Brasil tem apresentado grande desenvolvimento nas últimas décadas ${ }^{1}$, como resultado de políticas e linhas de financiamento que incentivaram a formação de pesquisadores e a consolidação de grupos de pesquisa, particularmente em instituições públicas de ensino superior e pesquisa. A saúde coletiva assume posição de destaque com crescimento sustentado a partir da segunda metade da década de 90 do século $\mathrm{XX}^{2}$. A epidemiologia tem importante contribuição no incremento da produção científica desta área ${ }^{2,3}$, especialmente entre aquela divulgada sob a forma de artigos científicos de periódicos nacionais e internacionais.

Entre os determinantes para essa tendência, verifica-se a expansão do ensino pós-graduado de epidemiologia, inserido em programas de mestrado e doutorado em saúde coletiva, que proliferaram particularmente a partir dos anos 90, em todo o Brasil, ainda que acompanhando a tendência de forte concentração no sudeste ${ }^{4,5}$. Essa expansão foi acompanhada de mudanças progressivas nas políticas de formação pós-graduada e no aperfeiçoamento do Sistema de Avaliação de Pós-Graduação pela Coordenação de Aperfeiçoamento de Pessoal de Nível Superior do Ministério de Educação (CAPES/MEC). O Sistema, criado em 1976, tem entre seus objetivos:

"estabelecer o padrão de qualidade exigido dos cursos de mestrado e de doutorado e identificar os cursos que atendem a tal padrão; (...) impulsionar a evolução de todo o Sistema Nacional de Pós-graduação, SNPG, e de cada programa em particular, antepondo-lhes metas e desafios que expressam os avanços da ciência e tecnologia na atualidade e o aumento da competência nacional nesse campo; contribuir para $o$ aprimoramento de cada pro- 
grama de pós-graduação, assegurando-lhe o parecer criterioso de uma comissão de consultores sobre os pontos fracos e fortes de seu projeto e de seu desempenho e uma referência sobre o estágio de desenvolvimento em que se encontra; contribuir para o aumento da eficiência dos programas no atendimento das necessidades nacionais e regionais de formação de recursos humanos de alto nível; (...)" (Capes/MEC. Avaliação da Pós-Graduação. Capturado em http:// www.capes.gov.br/avaliacao/ avaliacaopos.html em 3/9/2007)

A avaliação anual e trienal dos programas é efetuada por uma comissão em cada área e baseada em um conjunto de critérios que buscam aliar qualidade e eficiência. Em período recente, especialmente na década dos 2000, isso tem resultado na exigência cada vez maior de redução do tempo médio de titulação e no aumento da produção docente-discente veiculada em periódicos classificados pela relevância nacional e internacional.

Neste artigo, pretendo debater a proposta de "consórcio de pesquisas" do Programa de Pós-Graduação em Epidemiologia da Universidade Federal de Pelotas (PPGE/UFPel) $^{6}$, destacando algumas implicações pedagógicas deste modelo e apresentando em contraste a estratégia adotada pelo Programa de Pós-Graduação em Saúde Coletiva da Universidade Federal da Bahia (PPGSC/UFBA) para formação em pesquisa epidemiológica. Antes de fazê-lo, procuro contextualizar o debate situando-o brevemente no quadro da epidemiologia brasileira, suas principais características de desenvolvimento e pontuando o potencial dos inquéritos domiciliares como ferramenta de diagnóstico de saúde para subsidiar políticas públicas. Após discutir as principais vantagens e limites dos modelos adotados em dois importantes pólos de ensino e pesquisa epidemiológica no país, concluo levantan- do algumas questões que se colocam como desafios para a formação pós-graduada de pesquisadores na atualidade.

\section{Epidemiologia e formação em pesquisa}

Uma característica marcante da produção epidemiológica brasileira é sua firme inserção na área de saúde coletiva ${ }^{2,3}$ e sua orientação para problemas de grande relevância social, sendo reconhecida a participação de pesquisadores em foros e instâncias de formulação e monitoramento das políticas públicas de saúde no país.

Tendo nascido com a saúde pública, seu ensino formal teve início na década de 20 do século passado no Instituto de Higiene, hoje Faculdade de Saúde Pública da Universidade de São Paulo ${ }^{5}$. Mas foi a partir dos anos 80, com a constituição do campo da saúde coletiva e a criação da Associação Brasileira de Saúde Coletiva (Abrasco), que a expansão da epidemiologia brasileira vai se intensificar com esta característica de inserção na área, seja nos grupos de pesquisa, seja nos programas de pós-graduação. Como marco do papel impulsionador da Abrasco, destaca-se a criação de sua Comissão de Epidemio$\operatorname{logia}^{7}$, em 1984, responsável pela elaboração periódica de planos diretores (1989, 1994, 2000 e 2006), onde a formação em pesquisa e o fortalecimento de uma base institucional especializada têm constituído prioridades de ação. Os Congressos Brasileiros de Epidemiologia e a Revista Brasileira de Epidemiologia da ABRASCO refletem o grau de institucionalização da disciplina na área de saúde coletiva.

A epidemiologia brasileira tem também se caracterizado por sua estreita articulação com a prestação de assistência à saúde e o firme compromisso com a luta contra as desigualdades sociais. Neste contexto, os estudos transversais têm sido largamente utilizados na pesquisa epidemiológica nacional, buscando embasar políticas públicas voltadas à equidade e à justiça social. Têm sido freqüentemente adotados como estratégia de produção de da- 
dos para dissertações e teses nos programas de pós-graduação em epidemiologia. É sabido, entretanto, que grande parte investiga populações selecionadas, tais como escolares e usuários de serviços de saúde, o que aumenta a viabilidade das pesquisas, ao facilitar o acesso à população de estudo, mas incorre em vieses de seleção que limitam a generalização dos resultados e, muitas vezes, fornecem um diagnóstico imperfeito das necessidades de saúde.

Os inquéritos domiciliares com amostras representativas das populações apresentam vantagens para a produção de conhecimentos e para a formação de pesquisadores:

- São estratégia adequada ao diagnóstico de saúde e o levantamento de novas questões e hipóteses para estudos futuros;

- Permitem estimar parâmetros populacionais e identificar necessidades de saúde (e não apenas doenças) em populações sadias;

- Permitem a validação de instrumentos, propiciando a conclusão mais breve de estudos intermediários;

- Têm produção de dados relativamente rápida;

- A análise é relativamente mais simples do que em outros desenhos de estudo, o que favorece a iniciação em pesquisa.

Esse tipo de desenho é muito adequado, portanto, aos projetos de pesquisa que tenham entre seus objetivos a formação de estudantes em nível de mestrado, tanto pela simplicidade analítica quanto pelo tempo de duração.

\section{Consórcio de pesquisas: uma estratégia para a formação em Epidemiologia}

O “consórcio de pesquisa” do PPGE/ $\mathrm{UFPel}^{6}$ é uma estratégia de formação em pesquisa em nível de mestrado que, basicamente, reúne todos os alunos na realização de um único inquérito domiciliar, de onde se originam seus trabalhos de conclusão apresentados sob a forma de artigos.

Antes de tudo, é preciso destacar que o Centro de Pesquisas Epidemiológicas (UFPel) vem se constituindo em um pólo de produção de conhecimentos epidemiológicos com tradição de qualidade. Seu Programa de Pós-Graduação em Epidemiologia, com criação relativamente recente (mestrado acadêmico em 1991 e doutorado em 1998), apresentou consolidação rápida, atingindo nível de excelência e sendo atualmente um dos programas classificados em nível 6 pelo sistema de avaliação da Capes do Ministério da Educação.

Conforme pode ser confirmado em sua página na internet (http://www.epidemioufpel.org.br/_cpe/cpe.php), o Programa desde sua constituição baseou-se no propósito de "formar epidemiologistas que tivessem experiência de campo e soubessem não só analisar dados, mas também tivessem a capacidade de organizar e realizar levantamentos de dados na comunidade".

A proposta de "Consórcio de pesquisas” ${ }^{6}$ resulta do esforço de adequação às novas políticas de formação pós-graduada, buscando aliar qualidade e eficiência e assegurar experiência de pesquisa empírica e divulgação científica. Apresenta como principais vantagens a experiência de participação em pesquisa em todas as suas etapas e a oportunidade de realização de inquérito domiciliar. Pauta-se no aumento da eficiência e da produtividade, com diminuição do tempo médio de titulação e custo, além de impulsionar o aumento da publicação discente.

Como estratégia pedagógica, o Consórcio de pesquisas tem alguns limites que merecem ser discutidos. Em primeiro lugar, a escolha de tema é apenas o passo inicial para a formulação de questões de investigação, esta última sendo etapa crucial para o desenvolvimento de uma pesquisa. Constituindo um desafio mesmo para pesquisadores experientes, as questões de pesquisa resultam do amadureci- 
mento adquirido na maioria das vezes pelo trabalho coletivo organizado em grupos e linhas de pesquisa. Desse modo, a realização de projetos em consórcio estruturado com finalidade didática, de modo independente dos grupos de pesquisa do Centro de Pesquisas Epidemiológicas, priva os alunos de compartilharem o cotidiano de investigação de um dos principais pólos de produção de conhecimentos no país.

Um segundo limite a ser comentado diz respeito à elaboração de questionário no "consórcio de pesquisa" como mosaico de investigações, onde se estabelece um limite prévio de dez questões por aluno, com alternativa única de resposta. Isso compromete a contextualização do problema investigado e a análise das inter-relações com outros determinantes. Há também uma redução do social a indicadores socioeconômicos de caráter geral, que serão relacionados a desfechos - problemas de saúde ou uso de serviços -, independentemente do tipo de objeto.

Já que a cada turma realiza-se um novo inquérito, um aspecto não desprezível é o impacto potencial do excesso de questionários na população, o que não se limita ao aumento de recusas e perdas, mas pode influenciar a validade das informações prestadas.

O propósito do programa de assegurar o contato prático com a coleta de dados acaba se sobrepondo a todos os demais ao conferir ênfase excessiva a uma das etapas da pesquisa empírica. Subjacente a isso é passada a idéia de que o método se sobrepõe à teoria. Mais ainda, ao estruturar diagnósticos de saúde para viabilizar a prática dos mestrandos, ou seja, criando condições "artificiais" com finalidade didática, corre-se o risco de inculcar nos alunos a idéia de pesquisa meramente instrumental.

Cabe comentar os resultados alcançados na redução do tempo de titulação e no aumento da publicação discente, além da conclusão de que, adicionalmente, a iniciativa contribuiria para diminuir o custo da pesquisa. É preciso assinalar que em termos de avaliação de impacto, estamos comparando situações pré e pós-intervenção, mas sem grupo controle. A pressão institucional para redução do tempo de titulação tem promovido resultados que, na maioria dos programas de pós-graduação, adotam estratégias variadas. $\mathrm{O}$ aumento da produção discente - um pouco mais difícil de ser assegurada, exceto quando são adotados mecanismos que condicionam a aprovação dos alunos à publicação (ou pelo menos a aceitação) prévia ao momento da defesa - também tem sido alcançada pela co-autoria com orientadores e a inserção dos alunos em grupos de pesquisa. Quanto à diminuição do custo na presente iniciativa, pode-se argumentar que são comparadas duas situações extremas - o atual "consórcio de pesquisas" e o que ocorria anteriormente com a realização de projetos individuais, financiados pelos próprios alunos. Entre estas alternativas podem ser experimentadas outras que conjuguem maior racionalidade de recursos à inserção mais efetiva do corpo discente no processo regular de produção de conhecimentos científicos das instituições onde são sediados os programas de formação pós-graduada.

\section{Integrando pesquisa, ensino e cooperação técnica: a estratégia adotada no PPGSC/UFBA}

O PPGSC/UFBA teve seu início com a criação em 1973 do Mestrado em Saúde Comunitária; em 1989, implantou-se o Doutorado em Saúde Pública e em 2001 o Mestrado Profissionalizante ${ }^{8}$. É considerado um programa consolidado, com nível de excelência, tendo alcançado o grau A pelo Sistema de Avaliação da CAPES desde 1982, o nível 5 a partir de 1998 e 6 desde 2004. No período de 1973 a 2006, foram titulados 179 alunos de doutorado, 371 de mestrado acadêmico e 78 do mestrado profissional.

Tem como propósito a formação de pesquisadores, docentes e quadros técnicos, adotando uma perspectiva de ensino 
integrado à pesquisa e à cooperação técnica, por meio da incorporação dos alunos nos grupos de pesquisa do Instituto de Saúde Coletiva (ISC), onde está sediado. Seu projeto pedagógico enfatiza a natureza interdisciplinar da saúde coletiva, privilegiando a integração entre estudantes, pesquisadores e profissionais de distintas áreas do conhecimento e formação diversificada. O trabalho é organizado em linhas de pesquisa desenvolvidas pelos programas integrados de ensino, pesquisa e cooperação técnica que compõem a estrutura matricial do ISC.

O mestrado e o doutorado acadêmicos são oferecidos em três áreas de concentração: Epidemiologia, Planificação e Gestão em Saúde e Ciências Sociais em Saúde. Entretanto, os alunos das diferentes áreas, além de conteúdos específicos, compartilham disciplinas obrigatórias, para a constituição de uma base conceitual comum, que propicie o diálogo interdisciplinar pretendido. Entre elas, destaca-se a disciplina "Epistemologia e metodologia de pesquisa em saúde”, oferecida no primeiro semestre e obrigatória para o mestrado e o doutorado das três áreas de concentração. O programa combina aulas expositivas e oficinas para construção dos projetos dos alunos as quais podem ter recorte temático ou metodológico. As oficinas reúnem geralmente oito a dez alunos de mestrado e doutorado sob a coordenação de um docente que representa a área de concentração onde eles se inserem. O produto final a ser avaliado é o esboço do projeto de pesquisa apresentado por escrito e em sessão de pôster para a qual são convidados docentes, pesquisadores e alunos.

O PPGSC tem como atividade regular semanal uma Sessão de Pesquisa Orientada, onde são apresentados e debatidos pelos docentes, pesquisadores, alunos e especialistas convidados temas e trabalhos científicos da saúde coletiva, incluindo projetos e trabalhos de conclusão. Também são oferecidas oficinas de elaboração de projetos e artigos; no doutorado, inte- gram o currículo os seminários avançados que aprofundam aspectos teóricos, metodológicos e operacionais dos projetos de investigação.

Além de atividades curriculares, a principal estratégia pedagógica para formação em pesquisa é a inserção dos alunos nos programas integrados por meio de seus grupos de pesquisa. Isso visa atender ao pressuposto de que a produção de conhecimentos é um trabalho coletivo e uma prática social e histórica. Entende-se que a formação em pesquisa é um processo longo que inclui a transmissão de conhecimentos teóricos e metodológicos, mas também a inculcação de atitudes profissionais, políticas e éticas; ou seja, essa socialização necessariamente tem que se dar por meio do contato cotidiano com outros pesquisadores. Assim sendo, os projetos dos alunos devem integrar as linhas de pesquisa de seus orientadores e se articular a outros projetos de pesquisa, o que assegura sua viabilidade e a otimização de recursos materiais, humanos e simbólicos.

As implicações pedagógicas da inserção nos programas integrados podem ser grosso modo sumarizadas nas seguintes:

- Integração de pesquisadores, docentes e alunos de vários níveis e formação disciplinar;

- Formulação de questões de pesquisa e recortes de objetos em linhas de pesquisa;

- Adequação de teorias e métodos aos objetos;

- Exposição aos vários desenhos/estratégias de investigação;

- Compartilhamento da análise e interpretação de resultados em sessões científicas dos programas;

- Diálogo interdisciplinar com diferentes aproximações sobre um mesmo objeto;

- Integração de ensino, pesquisa e extensão.

Por meio do trabalho coletivo, pretende-se articular a produção científica à intervenção social, com vistas à aplicação do 
conhecimento produzido. A inserção dos alunos neste cotidiano permite o compartilhamento de valores e a formação de jovens pesquisadores que buscam aliar a qualidade técnico-científica, com a relevância social dos produtos e o compromisso ético-político de transformação social. Os alunos são instigados a refletir sobre aspectos éticos envolvidos na produção do conhecimento, desde a construção de seus projetos de pesquisa, submetidos para aprovação pelo Comitê de Ética do ISC, até o compromisso social com a publicação e divulgação dos produtos para a comunidade científica e para a sociedade. São estimulados a apresentar o trabalho final sob a forma de artigo (um para mestrado e três para doutorado), propiciando rápida divulgação dos resultados em revistas nacionais e internacionais.

Na última década, a perspectiva de redução do tempo médio de titulação e de consolidação das áreas de concentração dos cursos envolveu ajustes na estrutura curricular, as quais foram expressas no atual regimento do Programa, aprovado em 2000, onde fica definido 24 meses como o tempo de duração máxima do mestrado acadêmico e 48 meses o do doutorado. Isso é assegurado não apenas por medidas normativas mas pelo monitoramento permanente dos processos intermediários, tais como cumprimento de créditos e realização de exame de qualificação, até a finalização no momento da defesa de teses e dissertações.

\section{Comentários finais}

A pesquisa epidemiológica no Brasil tem se expandido, especialmente na última década, mantendo sua inserção na saúde coletiva e contribuindo para a formulação e o monitoramento de políticas públicas mais adequadas às necessidades de saúde da população. Um dos principais determinantes para esta tendência tem sido a formação de epidemiologistas em programas de pós-graduação, que se multiplicaram nas universidades públicas do país em forte articulação com o setor saúde.
A consolidação de um sistema nacional de avaliação da formação pós-graduada no país tem buscado assegurar a qualidade dos programas e contribuído para a maior eficiência na formação de recursos humanos de alto nível. Mudanças nos critérios de avaliação com ênfase na redução do tempo de titulação e no estímulo à publicação em periódicos de circulação internacional têm tido impacto na estrutura curricular, na duração dos cursos e nos produtos finais.

O surgimento de uma nova modalidade de curso - o mestrado profissionalizante - tem redefinido os propósitos de formação no mestrado acadêmico que, na área de saúde coletiva, e particularmente na epidemiologia, tradicionalmente formava pesquisadores, mas também cumpria a finalidade de formação de gestores e profissionais de saúde em busca de aprimoramento.

No cenário atual, algumas questões se colocam. O mestrado acadêmico é prioritariamente uma etapa da formação em pesquisa? E a formação em ensino?

A desvalorização crescente das atividades de ensino e da formação docente reflete-se não apenas na redução de espaço e do conteúdo na estrutura curricular dos cursos, como resposta à pressão para redução do tempo de titulação e à excessiva valorização do componente de produção e divulgação científica nos processos de avaliação pela Capes, mas também se expressa nos próprios critérios de avaliação da produção, que hierarquizam os produtos, atribuindo maior valor à publicação de artigos em periódicos de língua inglesa, menosprezando a produção de livros especialmente voltados à finalidade didática.

Ainda que se reserve ao mestrado acadêmico o papel de formação de pesquisadores, cabe perguntar se pesquisa epidemiológica é fundamentalmente a produção de dados primários. E a análise de dados secundários para a vigilância à saúde? A sofisticação de recursos de manuseio de grandes bases de dados, tais como técnicas de linkage, análise espacial e de séries 
temporais, tem colocado inúmeros desafios e ampliado as potencialidades de pesquisa avaliativa, com potenciais benefícios para a avaliação em saúde.

Por último, a experiência de pesquisa é necessariamente experiência de campo? É preciso envolver todas as fases da pesquisa para uma formação inicial?

Essas e outras questões requerem uma atualização do debate sobre a formação de epidemiologistas no país de modo a construir alternativas sólidas e adequadas à realidade nacional. Os desafios incluem o aprimoramento do sistema nacional de avaliação da formação pós-graduada, sem perder a perspectiva de fortalecimento da epidemiologia mantendo seus compromissos históricos com a saúde coletiva e com a aplicação dos conhecimentos para a transformação social.

\section{Referências}

1. Guimarães R. Pesquisa em saúde no Brasil: contexto e desafios. Rev Saúde Pública 2006, 40 (N Esp): 3-10.

2. Guimarães R,Lourenço R, Cosac S. A pesquisa em epidemiologia no Brasil. Rev Saúde Públ 2001; 35(4): 32140.

3. Barreto ML. Crescimento e tendência da produção científica em epidemiologia no Brasil. Rev Saúde Públ 2006; 40 (NEsp.): 79-85.

4. Hartz ZMA, Nunes TCM. Formação e capacitação de recursos humanos no Brasil: situação atual, desafios e perspectivas da pós-graduação em Saúde coletiva no Brasil. In: Leal MC, Freitas CM. Cenários possíveis: experiências e desafios do mestrado profissional na saúde coletiva. Rio de Janeiro: Editora Fiocruz; 2006. pp. 49-63.
5. Barata RB. Tendências no ensino da epidemiologia no Brasil. Rev Panam Salud Pública 1997, 2(5):334-41.

6. Programa de Pós-Graduação em Epidemiologia da UFPel. O mestrado em consórcio do Programa de PósGraduação em Epidemiologia da UFPel: uma experiência inovadora. Rev Bras Epidemiol (no prelo) 2007.

7. ABRASCO. 1986. I Reunião Nacional sobre Ensino e Pesquisa em Epidemiologia, Estudos de Saúde Coletiva. n. 4. pp. 93-105.

8. Dourado I, Teixeira CF, Aquino E, Silva LMV, Paim JS, Almeida Filho N. Mestrado Profissional em Saúde Coletiva: uma proposta alternativa para qualificação de docentes e técnicos em saúde - Instituto de Saúde Coletiva da UFBa (2001-2005). Rev Bras Pós-Graduação 2005; 2 (4): 61-71. 